\title{
VERFASSUNG UND POLITIK IN SENEGAL SEIT DER UNABHÄNGIGKEIT
}

\author{
Von Klaus Ziemer
}

Die Republik Senegal hat seit der Erklärung der Unabhängigkeit im Jahre 1960 mit Léopold Sédar Senghor bis heute denselben Politiker an der Spitze des Staates; die Regierungspartei „Union Progressiste Sénégalaise“ (UPS), 1960 „parti dominant" gegenüber mehreren Oppositionsparteien, ist heute die einzige legale Partei des Landes. Das Militär, das in einigen westafrikanischen Staaten nach einem Staatsstreich an der Macht ist, hat in Senegal nie einen Putschversuch unternommen. In auffallendem Kontrast zu dieser zumindest oberflächlichen Kontinuität stehen die zahlreichen Änderungen, denen die Verfassung seit 1960 unterworfen wurde und die fast durchweg das Resultat politischer Krisensituationen waren. Verändert haben sich im wesentlichen die von der Verfassung etablierten politischen Institutionen und ihr Verhältnis untereinander. Der Verfassungstext spiegelt jedoch nur bedingt die politische Realität wider, da im politischen System des Senegals zum einen die in der Verfassung nicht erwähnte UPS als "dominierende“ bzw. Einheitspartei eine wesentliche Rolle spielt und zum anderen die "modernen“ politischen Institutionen von Partei und Staat nur sehr zögernd den Bereich traditioneller politischer Kräfte absorbieren. Eine Analyse der institutionellen Änderungen seit der Unabhängigkeit hat daher neben dem Verfassungstext auch den soziopolitischen Kontext zu untersuchen, aus dem die einzelnen Verfassungsreformen hervorgegangen sind. Da Senegal als älteste französische Kolonie in Schwarzafrika auf eine lange Geschichte moderner politischer Institutionen zurückblickt ${ }^{1}$, soll vor der Behandlung der Verfassungen Senegals ab 1960 ein kurzer Abriß über deren Vorläufer und insbesondere über die Entwicklung ab 1958 stehen, die über die autonome Republik Senegal und die Mali-Föderation zur staatsrechtlichen Unabhängigkeit Senegals führte.

I.

Innerhalb des französischen Kolonialreichs in Schwarzafrika nahm Senegal bis zum Zweiten Weltkrieg eine Sonderstellung ein, da die Kolonie erstmals 1848 und 1849, dann ab 1871 bzw. 1879 regelmäßig einen Abgeordneten in die französische Nationalversammlung wählte. Aufgrund eines Gesetzes vom 24. 4.1848 und einer circulaire ministerielle vom 27. 4. 1848 galten die Einwohner der „vier Gemeinden“ (St. Louis, Rufisque, Gorée und Dakar) an der senegalesischen Küste als französische citoyens, was zu Beginn des 20. Jahrhunderts zwar wieder umstritten war, von dem 1914 gewählten ersten afrikanischen Abgeordneten in der französischen Nationalversammlung, Blaise Diagne, gegen die Rekrutierung von mehr als 100000 afrikanischen Soldaten im Ersten Weltkrieg in Gesetzen 1915 und 1916 jedoch endgültig gesichert wurde. 1879 wurde den vier Gemeinden nach dem Vorbild

\footnotetext{
1 Der vorliegende Beitrag beruht in einigen Teilen auf dem Artikel „Senegal ${ }^{\alpha}$ des in Vorbereitung befindlichen Handbuclis Franz Nuscheler/Klaus Ziemer: Politische Organisation und Repräsentation in Afrika, Berlin 1974 (= Band II aus der Reihe "Wahl der Parlamente", hrsg. von Dolf Sternberger, Bernhard Vogel, Dieter Nohlen und Klaus Landfried). Dort ist vor allem die Entwicklung bis zur Unabhängigkeit ausführlicher dargestellt.
} 
eines französischen Departements ein Conseil Général zugestanden, dessen Kompetenz mit der Umwandlung in einen "Conseil Colonial“ 1920 und der Ausdehnung der territorialen Zuständigkeit auf die ganze Kolonie allerdings eingeschränkt wurde. Bereits in den siebziger Jahren des 19. Jahrhunderts erhielten die „vier Gemeinden" volles französisches Kommunalrecht, und zwischen 1904 und 1925 wurden weitere 14 Gemeinden $\mathrm{zu}$ "Communes mixtes" erhoben². Zwar boten die Zusammensetzung der Wählerschaft - die „originaires“ (Afrikaner der „vier Gemeinden") waren ganz überwiegend Analphabeten - und die offene Stimmabgabe zahlreiche, bereits im 19. Jahrhundert genutzte Möglichkeiten, die Wahlen zu manipulieren ${ }^{3}$; im Vergleich zu den anderen französischen Territorien in Schwarzafrika nahm Senegal jedoch auf kommunaler und territorialer Ebene sowie durch die Wahl des Abgeordneten in die französische Nationalversammlung eine privilegierte Stellung ein.

Nach der Bildung der Volksfrontregierung in Frankreich wurde 1937 in Senegal ein Zweig der französischen SFIO gegründet, in der Afrikaner die Mehrheit stellten und in der von Anfang an an führender Stelle Lamine Guèye tätig war, der 1921 mit einer Arbeit über den Status der originaires in den „vier Gemeinden“ promoviert hatte. 1945/46 als Führer eines „Bloc Africain“ je mit überwältigender Mehrheit in die französische Nationalversammlung gewählt, erkannte Lamine Guèye nicht die Bedeutung der allmählichen Ausdehnung des Wahlrechts auf die Bevölkerung in ganz Senegal und beließ den Schwerpunkt der Partei in den vier alten Gemeinden. Léopold Sédar Senghor (geboren 1906), der bis 1945 in Frankreich als Studienrat gelehrt hatte, durch die Formulierung der „négritude“ ${ }^{4}$ bekannt geworden und von Lamine Guèye 1945 als „Abgeordneter für den Busch“ nach Senegal zurückgeholt worden war, gründete 1948 einen „Bloc Démocratique Sénégalais" (BDS), der von 1951 an alle Wahlen zur französischen Nationalversammlung und zum senegalesischen Territorialparlament gewann ${ }^{5}, 1957$ in den ersten Wahlen zur Territorialversammlung nach der Einführung der „Loi Cadre" 6 mit 47 von 60 Sitzen die absolute Mehrheit errang und mit dem Lehrer Mamadou Dia den ersten Regierungschef („vice-président du conseil“) im neuen Institutionensystem stellte. Anfang 1958 schloß sich die 1957 zum „Parti Sénégalais d’Action Socialiste" (PSAS) umgebildete Partei Lamine Guèyes der Regierungspartei an, die bereits mehrere kleinere politische Gruppierungen absorbiert hatte und sich nun als „Union Progressiste Sénégalaise“ neu konstituierte. Durch den Eintritt jüngerer, radikalerer Kräfte in die UPS erhielten nationalistische Strömungen einen stärkeren Auftrieb, und erst nach erbitterten Diskussionen und einem für de Gaulle in Senegal viel unfreundlicheren Empfang als in Guinea entschied die UPS-Führung, beim Referendum über die Verfassung der V. Französischen Republik am 28. 9. 1958 für ein „Ja“ zu plädieren. Gegen den Verfassungsentwurf — und damit für die sofortige Unabhängigkeit - sprachen sich neben einigen Gewerkschaften der

2 Jean-Paul Masseron: La place des communes dans les structures administratives sénégalaises et la réforme communale de 1966, in: Revue Juridique et Politique 21, 1967, S. 553.

3 B. Mille: The ${ }_{n}$ Black Vote ${ }^{\alpha}$ in Senegal, in: Journal of the African Society 1, 1901, S. 64-79, spricht von Senegal als einem protten borough"; zu den politischen Auseinandersetzungen in Senegal im 19 und zu Beginn des 20. Jahrhunderts siehe G. Wesley Johnson, jr.: The Emergency of Black Politics in Senegal. The Struggle for Power in the Four Communes 1900-1920, Stanford 1971.

4 Siehe dazu u. a. I. L. Markovitz: Léopold Sédar Senghor and the Politics of Négritude, London 1969.

5 Zur internen Organisation des BDS und zur Wahlkampfführung siehe Ruth Schachter Morgenthau: Political Parties in French-Speaking West Africa, Oxford 1964, S $146 \mathrm{ff}$.

6 Loi 56-619 vom 23. 6. 1956, JORF 24. 6. 1956, S. 5782, die vor allem allgemeines Wahlrecht, die Einführung einer von der Assemblée Territoriale gewählten Regierung und begrenzte Autonomie für die afrikanischen Territorien brachte. 
1957 von Majhemout Diop gegründete, auf Intellektuelle, vor allem Hochschulabsolventen, in den Städten beschränkte „Parti Africain de l'Indépendance“ (PAI) und der "PRA-Sénégal"7 aus, in dem sich Abdoulaye Ly, Assane Seck und andere prominente UPS-Mitglieder zusammenschlossen, die von Anfang an den Entwurf abgelehnt hatten. Nach der Annahme der Verfassung der V. Republik in Senegal mit 97,6 Prozent der gültigen Stimmen wurde das Territorium am 15. 11. 1958 durch Beschluß der Territorialversammlung autonome Republik innerhalb der Communauté Française.

Bei der Ausarbeitung der Verfassung des autonomen Senegal ergaben sich Schwierigkeiten in der Kompetenzabgrenzung insofern, als nicht nur die übergeordnete Verfassungsordnung der Communauté Française, sondern auch die gleichzeitig ausgearbeitete Verfassung der Mali-Föderation zu berücksichtigen waren. Um die durch die „Loi Cadre“ vorgezeichnete „Balkanisierung“ von Französisch Westafrika (AOF) in acht Einzelstaaten (Guinea war bereits am 2.10. 1959 für unabhängig erklärt worden) nicht endgültig Wirklichkeit werden zu lassen, waren die Regierungschefs von Senegal, dem Französischen Sudan, Dahomey und Obervolta Ende 1958 übereingekommen, einen Bundesstaat „Mali“ zu bilden. Obervolta und Dahomey, die der gemeinsamen Verfassung im Januar 1959 noch zugestimmt hatten, zogen sich jedoch kurz darauf auf Druck der französischen Regierung und des Regierungschefs der Elfenbeinküste, Houphouet-Boigny, wieder zurück, so daß Senegal und der Französische Sudan sich allein in der Mali-Föderation zusammenschlossen ${ }^{8}$. Nach der Präambel der Bundesverfassung vom 17. Januar 1959 stand die Föderation jedem autonomen westafrikanischen Staat zum Anschluß offen. Sitz der Bundesbehörden war Dakar. Die Bundesversammlung, in die jeder Mitgliedsstaat zwölf (nach der Reduzierung auf zwei Bundesstaaten durch Verfassungsänderung vom 22. 4. 1959: 20) Abgeordnete entsandte, die das jeweilige Territorialparlament auf fünf Jahre aus seiner Mitte wählte, sollte mit absoluter Mehrheit den Ministerpräsidenten wählen, der vom Vertrauen des Parlaments abhängig war. Die Gesetzgebungskompetenz des Parlaments, dessen Macht nach dem Vorbild des "rationalisierten Parlamentarismus" der V. Französischen Republik stark reduziert war, war auf einen Katalog der Bereiche von Bundesinteresse beschränkt, die in den drei Abschnitten „staatsbürgerliche Freiheiten“, kulturelle sowie wirtschaftliche und finanzielle Einheit zusammengefaßt waren.

Am 24. Januar 1959 nahm das Territorialparlament von Senegal, das sich zur Verfassunggebenden Nationalversammlung konstituiert hatte, die Verfassung der autonomen Republik Senegal an, nach der der Präsident des Ministerrats, der zugleich Staatschef war, vom Parlament mit absoluter, ggfs. im dritten Wahlgang mit relativer Mehrheit eingesetzt wurde. Der Kompetenzkatalog des Parlaments blieb auf die gewohnheitsrechtliche Gesetzgebung, die Wahlgesetzgebung für das senegalesische Parlament, die Organisation der inneren Sicherheit u. ä. sowie auf diejenigen Bereiche beschränkt, die von der Mali-Föderation delegiert wurden. Die Initiative für die Gesetzgebung wie für die Verfassungsrevision lagen sowohl bei der Regierung als auch beim Parlament, wobei ein Antrag auf Verfassungsänderung von mindestens einem Drittel der Abgeordneten gestellt und von mindestens

7 Die UPS bildete seit 1958 die senegalesische Sektion eines interterritorialen „Parti du Regroupement

8 Zur Geschichte der Mali-Föderation siehe W. J. Foltz: From French West Africa to the Mali Federation, New Haven/London 1965. 
drei Fünfteln der Abgeordneten gebilligt werden mußte. Würde dieses Quorum nicht, wohl aber eine absolute Mehrheit erreicht, sollte ein Referendum entscheiden.

Nach den Parlamentswahlen vom März 1959, die der UPS bei 682865 von 832812 gültigen Stimmen ${ }^{9}$ aufgrund des Wahlgesetzes ${ }^{10}$ alle 80 Sitze sicherten, und der Konstituierung der Bundesinstitutionen (Modibo Keita aus dem Sudan Regierungschef, Mamadou Dia Vizepräsident des Ministerrats und L. S. Senghor Präsident der Bundesversammlung) gelang es Modibo Keita und Mamadou Dia im Dezember 1959, de Gaulles Zustimmung für die Unabhängigkeitserklärung von Mali zu erhalten. Nach einer Verfassungsänderung ${ }^{11}$ erhielt die Mali-Föderation am 20. Juni 1960 ihre staatsrechtliche Unabhängigkeit, doch dauerte ihre Existenz nur zwei Monate. Sie scheiterte an dem gegensätzlichen sozioökonomischen Unterbau der politischen Strukturen in beiden Bundesstaaten; Meinungsverschiedenheiten über die Außenpolitik, besonders über das Verhältnis zu Frankreich; ideologischen Differenzen zwischen den Parteiführungen von RDA-Sudan und UPS, die trotz Zusammenschluß in einem "Parti de la Fédération Africaine" (PFA) 1959 auf Landesebene weiterbestanden; Versuchen der sudanesischen Parteiführer, dem PFA auch in Senegal die disziplinierte und fast alle Bereiche des Staatswesens erfassende Organisation des RDA aufzuprägen; schließlich Differenzen über die Kandidatur Senghors für die Präsidentschaft der Republik Mali'².

Die gesetzgebende Versammlung Senegals, die am 20. August 1960 das Ende der Mali-Föderation erklärte und die Unabhängigkeit Senegals proklamierte, nahm entsprechend Art. 46 der senegalesischen Verfassung vom 24. 1. 1959, die dem Parlament bei Drei-Fünftel-Mehrheit verfassungsändernde Kompetenz einräumte, bereits am 26. 8. 1960 einstimmig den Entwurf einer Verfassung $a^{13}$, der Senegal eine dualistische Exekutive gab. Der Staatspräsident wurde auf sieben Jahre von einem „Kongreß“ gewählt, der sich — ähnlich wie der Wahlkörper bei Präsidentschaftswahlen in der ursprünglichen Verfassung der V. Französischen Republik aus der Nationalversammlung und je einem Mitglied der sieben Regional- und der Munizipalräte zusammensetzte. Der Präsident führte den Vorsitz im Ministerrat, war Chef der Streitkräfte, ernannte die höchsten Beamten und designierte den Ministerpräsidenten, der von der Nationalversammlung nach Vorlage seines Regierungsprogramms in offener Abstimmung mit absoluter Mehrheit gewählt wurde. Die Mitglieder der Regierung durften zwar dem Parlament angehören, ihre Funktion war jedoch unvereinbar mit dem Amt des Präsidenten der Nationalversammlung und der Zugehörigkeit zum Büro oder einer der Kommissionen des Parlaments. Die Nationalversammlung besaß die Gesetzgebungskompetenz innerhalb eines genau definierten Zuständigkeitskatalogs und konnte die Regierung

\footnotetext{
9 Detaillierte Ergebnisse nach Wahlkreisen bei Mamadou Lô: L’Union Progressiste Sénégalaise, in: Traoré, Lô, Alibert: Forces politiques en Afrique Noire, Paris 1966, S. 204.

10 Loi 59-004 vom 26. 1. 1959, JORS 19. 2. 1959, S. 146.

11 Loi organique 60-11 vom 18. 6. 1960, JORM vom 25. 6. 1960; an der Spitze der Exekutive stand nun ein Staatspräsıdent, der von einer gemeinsamen Versammlung der Parlamente des Bundes und der Bundesstaaten auf fünf Jahre gewählt werden sollte. Er nominierte den Regierungschef, der mit absoluter Mehrheit vom Bundesparlament zu wählen und von seinem Vertrauen abhängig war. In das Bundesparlament delegierte jeder Gliedstaat nach bisherigem Modus 20 Abgeordnete.

$12 \mathrm{Zu}$ einer detallierten Analyse der Spannungen zwischen den beiden Parteiführungen und zum Ablauf der Ereignisse siehe W. J. Foltz (Anm. 8); ferner u. a. François Zuccarelli: Un Parti Politique Africain: L'Union Progressiste Sénégalaise, Paris 1970, S. $69 \mathrm{ff}$.

13 Loi 60-45 vom 26. 8. 1960, JORS vom 31. 8. 1960; abgedruckt u. a. in: Revue Juridique et Politique 15, 1961, S. 453; D. G. Lavroff/G. Peiser: Les constitutions africaines, Bd. 1, Paris 1961, S. 190; L. Muracciole: Les constitutions des états africains d'expression française, Paris 1961, S. 124; Notes et Etudes Documentaires Nr. 2754 vom 22. 1. 1961, S. 42.
} 
durch Mißtrauensvotum stürzen. In Präzisierung des aufgrund der schnellen Redaktion ungenauen Textes von 1960 bestimmte ein Verfassungsgesetz von 196114, daß die Regierung nach zwei erfolgreichen Mißtrauensanträgen innerhalb von 18 Monaten nach Rücksprache mit dem Präsidenten der Republik und vor Entgegennahme des Rücktrittsgesuchs durch den Präsidenten die Nationalversammlung auflösen konnte, ausgenommen während der ersten 18 Monate der Wahlperiode des Parlaments. Die Bestimmungen über eine Verfassungsänderung blieben ähnlich wie in der bisherigen Verfassung. Nachdem L. S. Senghor Anfang September 1960 einstimmig zum Staatspräsidenten gewählt worden war, investierte die 1959 gewählte Gesetzgebende Versammlung, die sich zur Nationalversammlung von Senegal konstituiert hatte, Mamadou Dia als Ministerpräsidenten.

Die erste Verfassung des unabhängigen Senegal führte somit ein an das parlamentarische Regierungssystem angelehntes Institutionensystem ein, in dem ein Gleichgewicht zwischen Exekutive und Legislative angestrebt wurde. Vor dem Hintergrund der Verfassungen der übrigen 1960 für unabhängig erklärten Staaten von AOF und AEF zeichnet sie sich durch das Fehlen einer starken Exekutive aus, was sich in der Verteilung der exekutiven Kompetenzen auf Staats- und Regierungschef sowie - trotz im übrigen deutlicher Anlehnung an die Verfassung der V. Französischen Republik - dem Fehlen von Notstandsvollmachten für den Staatspräsidenten ausdrückte. Die Lösung einer dualistischen Exekutive bot zugleich die Möglichkeit, den seit 1957 amtierenden Regierungschef Mamadou Dia im Amt zu belassen und den beiden führenden Persönlichkeiten der UPS annähernd gleichrangige Funktionen im Staat zuzuweisen. Der Hauptrepräsentant des ehemaligen PSAS in der UPS, Lamine Guèye, war bis zu seinem Tode 1968 Präsident der Nationalversammlung.

\section{II.}

Der soziopolitische Kontext, für dessen Entwicklung durch die neue Verfassung der institutionelle Rahmen abgesteckt war, war auf der Ebene der politischen Parteien durch die faktische Alleinherrschaft der UPS gekennzeichnet, die bei Fortbestehen der bisherigen Oppositionsparteien (PRA-Sénégal, PSS (s. u.), PAI ${ }^{15}$ ) als parti doninant alle wichtigen Positionen in Parlament und Regierung besetzte. Ener Kontrolle der Partei entzogen sich jedoch die islamischen Würdenträger, die auf die Masse der Landbevölkerung - trotz der mit ca. 25 Prozent höchsten Urbanisierungsquote im ehemaligen AOF lebt dort noch immer die überwiegende Mehrheit der Bevölkerung - einen stärkeren Einfluß besaßen als die modernen Institutionen von Partei und Staat ${ }^{16}$. Die "neuen Eliten “ in den Städten (Hochschulabsolventen, Beamte, Gewerkschaften etc.) standen der UPS zum großen Teil skeptisch gegenüber, und der moderne Sektor der Wirtschaft wurde fast vollständig von ausländischem, überwiegend französischem Kapital kontrolliert. Senegal war ferner durch eine hohe Analphabetenquote, die Erdnuß-Monokultur und eine totale ikonomische Dependenz von Frankreich gekennzeichnet. Zur Zeit der

\footnotetext{
14 Ioi organique 61-63 vom 12. 11. 1961, JORS 14. 11. 1961

15 Unruhen bei den Kommunalwahlen in St. Louis nahm die Regierung 1960 zum Vorwand, den PAI, der eine orthodox-kommunistische Politik Moskauer Richtung vertritt, zu verbieten; die zahlenmäßig allerdings sehr klcine Partei arbeitet seitiker im Untergrund.

16 Vgl. J. Paquet: Essai sur la tentative d'édification de la nation sénégalaise 1957-1963, Paris (Mémoire DES Science Politique, Faculté de Droit ct des Sciences Economiques) 1967 (hekt.), S. 73 f.
} 
Unabhängigkeit besuchten von 450000 Kindern im schulpflichtigen Alter nur 125500 (28 Prozent) Primär- und 9385 Sekundarschulen ${ }^{17}$, wobei innerhalb des Staatsgebiets beträchtliche Disproportionen zwischen den städtischen Zonen und dem Busch bestanden (1963: 75 Prozent Scholarisationsrate in der Region des Cap Vert (Dakar), 25 Prozent im Osten Senegals). An der Ende der fünfziger Jahre gegründeten Universität Dakar studierten 1963/64 erst 500 Senegalesen ${ }^{18}$, zu denen noch eine Anzahl von Studenten im Ausland, besonders in Frankreich, hinzukam. Die Folge war ein Mangel an qualifizierten afrikanischen "Cadres" im öffentlichen und privaten Bereich in den mittleren und gehobenen Laufbahnen, deren Plätze daher z. T. noch lange Jahre nach der Unabhängigkeit von Franzosen eingenommen wurden. Der Vorsprung der urbanisierten Küstenregionen gegenüber dem Rest von Senegal reflektierte sich auch in der ethnischen Zusammensetzung der Nationalversammlung: Die in der Gegend von Dakar konzentrierten Lebou (2 Prozent der Gesamtbevölkerung) stellten 13 Prozent der Abgeordneten, die 16 bzw. 15 Prozent der Bevölkerung umfassenden, aber in ländlichen Gegenden lebenden Serer und Fula je nur 6 Prozent $^{19} .90$ Prozent der aktiven Bevölkerung sind in der Landwirtschaft beschäftigt ${ }^{20}$, deren Anteil am BIP 1959 mit 43 Prozent weit vor Handel (26 Prozent) und Nahrungsindustrie (8 Prozent) lag21. Der Wert der 1959 kommerzialisierten Produktion in der Landwirtschaft (einschließlich Viehzucht) betrug 18564 Mill. F CFA, von denen 15120 Mill. auf die Erdnußproduktion entfielen ${ }^{22}$. Die Entwicklung der Erdnuß zur Monokultur war erst in den letzten Jahrzehnten von der Kolonialmacht forciert worden. Noch 1936/37 hatte der Anteil der Erdnuß an der gesamten landwirtschaftlichen Nutzfläche 48 Prozent, der der Hirse 45 Prozent betragen; bis 1960/ 61 hatte sich das Verhältnis auf 64,5 Prozent zu 29,1 Prozent verschoben ${ }^{23}$, so daß Senegal auf die Einfuhr von Lebensmitteln angewiesen ist, obwohl bei entsprechender Verbesserung der Infrastruktur eine Vielzahl von landwirtschaftlichen Produkten (Reis, Zuckerrohr, Gemüse u. a.) in großem Umfang angebaut werden könnte ${ }^{24}$. Ókonomisch blieb Senegal trotz der staatsrechtlichen Unabhängigkeit völlig von Frankreich abhängig; 1960 wurden bei einem Gesamtimport im Umfang von 42,5 Mrd. F CFA aus Frankreich Waren im Wert von 28,6 Mrd. importiert, und bei einem Exportvolumen von 27,9 Mrd. F CFA nahm Frankreich Waren im Wert von 23,0 Mrd. F CFA auf25. Die Bildung senegalesischer Gesellschaften im Bereich der Kommerzialisierung der Erdnuß-Produktion, des Kreditwesens u. a. nach 1960 änderte kaum etwas an der übermächtigen Stellung französischer Unternehmen im Großhandel und Kreditwesen und von Libanesen und Syrern im mitt-

17 Le premier plan de développement de la République du Sénégal, Notes et Etudes Documentaires Nr. 2911, 6. 8. 1962, S. 38.

18 Die letzten Zahlenangaben nach P. F. Gonidec: La République du Sénégal, Encyclopédie politique et constitutionneile, Paris 1968, S. 15.

19 Michael Crowder: Senegal. A Study of French Assimilation Policy, London 1967, S. 106.

20 P. F. Gonidec (Anm. 18), S. 12.

21 Zahlen nach Abzug der indirekten Steuern; Quelle: L'évolution économique du Sénégal, Notes et Etudes Documentaires Nr. 3054, 13. 1. 1964, S. 45. In dem offiziösen Bericht „République du Sénégal, Comptes éconorniques, années 1959, 1960,1961, 1962 ${ }^{\alpha}$ von Sérigne Diop, Oumar Thiaw, Bernard Mas, o. O., Dezeniver 1963, S. 10, wird, ebenfalls nach Abzug der indirekten Steuern für 1959 folgendermaßen aufgeschlüsselt: Landwirtschaft 44 Prozent, Industrie, Hochbau und öffentliche Bauarbeiten, Handwerk: 18 Prozent, Transport, Handel, andere Dienstleistungen: 38 Prozent.

22 NED 2911 (Anm. 17), S. 18.

23 Valy-Charles Diarassouba: L'évolution des structures agricoles du Sénégal. Destructuration et restructuration de l'économie rurale, Paris 1968, S. $121 \mathrm{f}$.

$24 \mathrm{Vgl}$. Samir Amin: L'Afrique de l'Ouest bloquée. L'économie politique de la colonisation 1880-1970, Paris 1972, S. $37 \mathrm{ff}$

25 NED 2911 (Anm. 17), S. 10. 
leren Handel26. Noch Ende der sechziger Jahre hatten die 1,5 Prozent der Bevölkerung zählenden Ausländer einen Anteil von 23 Prozent an den Einkommen und von 25 Prozent am Konsum im gesamten Senegal27. Auf der Ebene direkter politischer Einflußnahme reflektierte sich die Bedeutung der ausländischen Wirtschaftskreise darin, daß Franzosen ${ }^{23}$ ständig in der Nationalversammlung vertreten waren und bis heute mindestens eine Schlüsselstellung im Kabinett (Wirtschafts-, Finanz- oder Innenministerium) innehatten.

Bei der relativ niedrigen Zahl der Beschäftigten im sekundären und tertiären Wirtschaftsbereich ${ }^{29}$ und ihrer Beschränkung auf die urbanisierten Regionen ${ }^{30}$ waren die verschiedenen Gewerkschaftszentralen nur in den Städten verankert. Die Gewerkschaften von Senegal hatten jedoch die höchste Mitgliederzahl von ganz $\mathrm{AOF}^{31}$, standen aber immer in Distanz zum BDS; auch nach der Fusion der meisten Parteien zur UPS blieben wichtige Gewerkschaftszentralen mit der Opposition liiert ${ }^{32}$. Die UPS hat die Organisationen der Arbeitnehmer bis heute nicht völlig unter ihre Kontrolle bringen können. Die von ihr zu Beginn der sechziger Jahre als dominierende Gewerkschaftszentrale aufgebaute „Union Nationale des Travailleurs Sénégalais“ (UNTS) ${ }^{33}$ löste sie zu Beginn der siebziger Jahre wieder auf und versuchte, sie durch eine neue, der Partei direkt unterstellte Gewerkschaftsorganisation zu ersetzen (s. u.). Der tatsächliche Einfluß der Gewerkschaften übersteigt die relativ geringe Mitgliederzahl bei weitem. Die latente Opposition zur Regierung äußerte sich jedoch in manifester Weise erst seit Ende der sechziger Jahre.

$\mathrm{Zu}$ den verschiedenen Gruppen des Islam (Tidjaniya, Mouriden, Quadriya) bekennen sich über 90 Prozent der Bevölkerung ${ }^{34}$. Den geistlichen Führern gelang es in vielen Gegenden, in einem allmählichen Prozeß auch die Funktionen der traditionellen Chefferie zu übernehmen und ihre eigene Hierarchie auch im weltlichen Bereich zu etablieren. Ókonomisch und politisch einflußreich ist dabei vor allem der erst Ende des 19. Jahrhunderts gegründete $Z$ weig der Mouriden, bei denen die talibé (Gläubigen) dem jeweiligen Marabut völlig ergeben sind und Arbeit und Geschenke für ihn als heilsnotwendigen Teil der Religion betrachten. Da die Mouriden besonders im Hauptanbaugebiet von Erdnuß, den Regionen SineSaloum und Diourbel, ihren Rückhalt haben, kontrollieren ihre Führer einen beträchtlichen Teil der Erdnußproduktion ${ }^{35}$. Darüber hinaus besaßen die Chefs der islamischen Gruppen ein erhebliches politisches Gewicht, da sie als „grands électeurs“, denen eine persönliche Klientel ergeben war, über eine bedeutende Zahl

26 Siehe Samir Amin: Le Monde des Affaires Sénégalais, Paris 1969, passim.

27 Majhemour Diop: Histoire des classes sociales dans l'Afrique de l'Quest. 2. Le Sénégal, Paris 1972, S. 227.

$28 \mathrm{Daß}$ einige Franzosen nach der Unabhängigkeit die senegalesische Staatsbürgerschaft annahmen, stellt einen rein formalen Akt dar.

29 Exakte Zahlen sind nur schwer erhältlich; Diop (Anm. 27) schätzt die Zahl aller Lohnempfänger für 1960 auf 110000 ; die Zahl der offiziell registrierten Lohnempfänger betrug 196269939 (ebd.).

30 Diarassouba (Anm. 23), S. 177 bezitfert die 1959 dauernd oder zeitweilig gegen Lohn in der Landwirtschaft beschäftigten Arbeiter auf rund 12000 .

31 Franz Ansprenger: Politik im Schwarzen. Afrika, Köln/Opladen 1961, gibt im Anhang in Tabelle 5 die Zahl der Mitglieder aller Gewerkschaften in Senegal für 1955 mit 55700 an.

32 Vgl. u. a. Bakary Traoré: L'évolution des partis politiques au Sénégal depuis 1946 (Anm. 9), S. 92.

33 Einzelne Stufen von Fusionen und weiteren Absplitterungen siehe bei Zuccarelli (Anm. 12), S. 298 ff. Dort auch weitere Literatur.

34 Nach der Enquête démographique von 1960/61: 2780600 von 3049560 Einwohnern; Zahlen bei Zuccarelli (Anm. 12), S. 316.

35 Donal Cruise O'Brien: The Mourides of Senegal. The Political and Economic Organization of an Islamic Brotherhood, Oxford 1971, S. 216, Tabelle XXII, gibt an, daß 1957/58 die 18,5 Prozent der Bevölkerung umfassenden Mouriden einen Anteil von 24,6 Prozent an der senegalesischen Erdnußernte hatten. Nach D. G. Lavroff: La République du Sénégal, Paris 1966, S. 70, produzieren die Mouriden fast die Hälfte der senegalesischen Erdnußernte. 
von Wählerstimmen verfügten. Hatten die Marabuts vor dem Zweiten Weltkrieg bei nur wenig Gefolgschaft in den Städten an der Küste (und entsprechend wenig disponiblen Wählern) den Wahlkampf einzelner Kandidaten für die französische Nationalversammlung mitfinanziert, um gegen Repressionen der französischen Verwaltung gesichert $\mathrm{zu} \operatorname{sein}^{36}$, so zahlten mit der allmählichen Ausbreitung des allgemeinen Wahlrechts nach 1945 politische Gruppierungen, deren Organisationsnetz noch durchweg schwach war und die in den "grands électeurs" ein sicheres Mittel für die Gewinnung von Wählerstimmen besaßen, ihrerseits z. T. erhebliche Zuwendungen an die Führer islamischer Bruderschaften, deren Einfluß L. S. Senghor in hohem Maße seine Siege über Lamine Guèye in den Wahlen der fünfziger Jahre zu verdanken hatte ${ }^{37} .1958$ hatte die Haltung bedeutender Oberhäupter islamischer Gruppen offenbar großen Einfluß auf die erst spät gefaßte Zustimmung der UPS zum Verfassungsentwurf für die V. Französische Republik; die Marabuts hatten erklärt, bei einem negativen Ausgang des Referendums könne die Bevölkerung, die sie vertreten, darum ersuchen, mit Frankreich vereint zu bleiben ${ }^{33}$. Ein im November 1959 gegründeter "Conseil Supérieur des Chefs Religieux" brach 1959 wieder auseinander, nachdem ein Teil seiner Mitglieder unter Führung des Tidjaniya-Marabuts Ibrahima Niasse in einem Telegramm an de Gaulle dagegen protestierte, daß die senegalesische Verfassung von 1959 nur vom Parlament und nicht durch Referendum (bei dem die Marabuts ihren Einfluß für eine Ablehnung der Verfassung hätten geltend machen können) angenommen worden war. Die islamischen Führer hatten gefordert, ihrem Conseil solle in der Verfassung das Recht garantiert werden, die Hälfte der 80 Parlamentsabgeordneten zu ernennen ${ }^{39}$. Einige Marabuts unter Führung von Cheikh Tidjane Sy gründeten Anfang 1959 mit Unterstützung rechtsstehender Franzosen einen „Parti de la Solidarité Sénégalaise" (PSS), der versuchte, eventuell die Autonomie des Territoriums rückgängig zu machen und Senegal als überseeisches Departement weiter bei Frankreich zu belassen. Nach den Wahlniederlagen von 195940 löste sich die Gruppierung bis 1960 auf; ihre Führer zogen sich entweder aus der Politik zurück oder schlossen sich der UPS $a^{41}$.

Eine offene Einmischung der Marabuts in die Politik zuungunsten der Regierung unterblieb in der Zeit danach, doch stellten die Chefs der islamischen Gruppen aufgrund ihrer großen Klientel zumindest so lange eine potentielle Gefahr für die Regierung dar, als mehrere politische Parteien vorhanden waren (bis 1966) und die Marabuts bei der relativ schwachen Organisation der UPS ihre Wählerstimmen den Oppositionsgruppen zur Verfügung stellen konnten. Auch in der Zeit danach war die UPS-Führung in dem Maße auf die Kooperation der islamischen Würdenträger angewiesen, als deren Einfluß auf lokaler Ebene erheblich stärker als der von Staat und Partei war und die UPS bzw. ihre Führung Krisen durchliefen

36 Für den Wahlkampf von 1914 beschrieben bei G. Wesley Johnson, jr.: The Ascendancy of Blaise Diagne and the Beginning of African Politics in Senegal, in: Africa, Bd. 36, Nr. 3, Juli 1966, S. 240 ff.; ferner: Cruise O'Brien (Anm. 35), S. 264 f.

37 Cruise O’Brien (Anm. 35), S. 263; Schachter-Morgenthau (Anm. 5), S. 147 f. Da Senghor als Katholik gegenüber dem Moslem Lamine Guèye im Wettbewerb um die Gunst der islamischen Würdenträger benachteiligt war, setzte er sehr früh Mamadou Dia als Kontaktmann ein, der als Schüler der Marabuts deren Vertrauen genoß (ebd., S. 148).

38 Lavroff (Anm. 35), S. 37; Zuccarelli (Anm. 12), S. 323.

39 Cruise O'Brien (Anm. 35), S. 272.

40 Bei 99330 von 682865 gültigen Stimmen aufgrund des Wahlgesetzes kein Sitz; Zahlen nach Lô (Anm. 9), S. 204.

$41 \mathrm{Vgl}$. Ansprenger (Anm. 31), S. 338; Christian Coulon: Pouvoir politique et Pouvoir maraboutique au Sénégal, in: Année Africaine 1971 (Paris 1972), S. 157 ff.; ferner: Lucy C. Behrman: Muslim Brotherhoods and Politics in Senegal, Cambridge/Mass., 1970, S. $93 \mathrm{ff}$. 
(besonders ab 1968). Das Wohlverhalten der Chefs des Islam wurde kompensiert durch erheblichen Einfluß der Marabuts auf die Gesetzgebung (besonders im Bereich des Zivilrechts und der Agrargesetzgebung) und massive materielle Zuwendungen ${ }^{42}$.

Theoretische Grundlage der Innen- und Außenpolitik Senegals bildete das Postulat eines "senegalesischen Weges" zum afrikanischen Sozialismus"3, das Senghor in einer Synthese aus wissenschaftlichem Sozialismus, dem Evolutionsmodell Pierre Teilhard de Chardins und traditionellen Werten schwarzafrikanischer Kultur (wie Senghor sie in der „Négritude“ zu fassen suchte) formulierte, wobei er sich im ökonomischen Bereich auf die Theorien von François Perroux und Louis J. Lebret stützte ${ }^{44}$. Da die „freiwillige“ Teilnahme aller Betroffenen als wesentliche Voraussetzung für das Gelingen des Wirtschaftsplanes gilt, wurde auf die Nationalisierung der Produktionsmittel (außer den Eisenbahnen und den anderen Transportmitteln) verzichtet. Innerhalb der Ziele der Vierjahres-Pläne (1. Plan: 1961-1964) versuchte die Regierung, möglichst viel privates und ausländisches Kapital für Investitionen einzusetzen. Vor allem in der Landwirtschaft wurden Genossenschaften gegründet, für die ein ganzes Netz von Hilfsorganisationen im Bereich der Selbstverwaltung der Genossenschaften, der animation rurale, der Finanzierung und der Kommerzialisierung der Ernte etc. aufgebaut wurde ${ }^{45}$. Für die praktische Ausführung der von Senghor entworfenen allgemeinen Konzeption war Regierungschef Mamadou Dia verantwortlich, der auf eine forcierte Ausdehnung des „sozialistischen" Sektors in der Landwirtschaft drängte.

\section{III.}

Innerhalb der UPS machte sich der in der zweigleisigen Exekutive angelegte "natürliche“ Dualismus zwischen L. S. Senghor und Mamadou Dia in einer verstärkten Aktivität der "clans politiques“ bemerkbar. Clans, informelle Gruppierungen um eine Persönlichkeit, die politischen Einfluß zu gewinnen sucht ${ }^{46}$, hatten in der senegalesischen Politik zunächst als Basis von Komitees zur Unterstützung von Kandidaten bei Wahlen seit Anfang des Jahrhunderts eine bedeutende Rolle gespielt. Sie bildeten sich innerhalb der UPS auch um die beiden Spitzenpolitiker. Dia gelang es dabei offensichtlich, persönliche Kontrolle über den Verwaltungsapparat zu gewinnen. Senghor warf ihm nach dem Eklat Ende 1962 vor, er habe die Verwaltung nicht in den Dienst des Staates, der Nation oder der Partei, sondern seines persönlichen Clans gestellt ${ }^{47}$. Der sich Ende 1962 entladende Konflikt zwischen beiden Parteiführern hatte sich seit Monaten vor allem in personellen Auseinandersetzungen innerhalb der Partei angekündigt. Dia, der sich immer mehr an Widerstand gegen seine Politik stieß, regierte zunehmend autoritär

42 Insbesondere bei de! Produktion und Vermarktung von Erdnuß, vgl. Zuccarelli (Anm. 12), S. 325 f.

43 Die Terminulogie ist schwankend. Der Ausdruck "Senegalesischer Weg zum afrikanischen Sozialismus ${ }^{\alpha}$ wurde in Anlehnung an Peter Doll: Der senegalesische Weg zum afrikanischen Sozialismus, Hamburg 1966, gewählt.

$44 \mathrm{Zu}$ den theoretischen Grundlagen siehe Markovitz (Anm. 4), Kapitel V: The Definition of Senegalese Socialism, S. $119 \mathrm{ff}$

45 Vgl. Marguerite Camboulives: L'organisation coopérative au Sénégal, Paris 1967, S. 119 ff. und S. 261 ff. sowie H. de Decker: Le développement communautaire. Une stratégie d'édification de la nation, Paris/Den Haag 1967, S. $253 \mathrm{ff}$.

46 Bei den Wolof bilden sich clans oft um islamische Führer, bei den Tukulör im Norden auf Kastenbasis, bisweilen reflektieren die clans einen gewissen Generationenkonflikt, beruhen jedoch häufig auf rein persönlichen Rivalitäten.

47 So L. S. Senghor in der Neujahrsansprache am 1. 1. 1963, zitiert bei Ladipo Adamolekun: Bureaucrats and the Senegalese political process, in: Journal of Modern African Studies 9, 1971, S. 547. 
und befürwortete auch gegenüber der noch bestehenden Opposition einen härteren Kurs, während Senghor für die Aufnahme des oppositionellen „Bloc des Masses Sénégalaises" (BMS, s. u.) in die UPS plädierte. Der eigentliche Kern des Gegensatzes zwischen Senghor und Dia lag jedoch in der nie eindeutig geklärten Frage, worauf sich die Legitimität des Regimes letztlich gründe. Während Senghor immer wieder die Vorherrschaft der Staatsinstitutionen über die Partei betonte ${ }^{48}$, galt für Dia die Partei als oberste Instanz, der sich die Staatsinstitutionen unterzuordnen hatten. Für Senghor stellte sich das Verhältnis zwischen Verfassung und Partei nicht in Konkurrenz, sondern in hierarchischem Bezug dar; für Dia war es in einer komplexeren, "afrikanischen" Konzeption mit moralischen Kriterien verwoben $^{49}$ : „Dans le cas où il y a opposition entre le Droit et la Morale, je préfère rester du côté de la morale, car il n'y a possibilité d'existence de l'Etat sans éthique, sans respect réciproque des engagements". Als daher am 14. 12. 196239 der 80 Abgeordneten der Nationalversammlung einen Mißtrauensantrag gegen die Regierung Dia einbrachten, in dem in scharfen Worten Dias autoritärer Regierungsstil und das Beschneiden von Kompetenzen der Nationalversammlung durch die Regierung kritisiert wurde, und das Parlament am 17. 12. dem Antrag stattgab, entsprach dies zwar der verfassungsmäßigen Prozedur ${ }^{50}$, doch bestritt Dia die Legitimität des Vorgehens, da nach einem Beschluß des Parteitages von Thiès (4. bis 6. 2. 1962) die Kontrolle von Regierung und Parlament der Partei unterworfen war und der Mißtrauensantrag zunächst vom Bureau politique der UPS hätte gebilligt werden müssen. Für Senghor waren Regierung und Abgeordnete jedoch trotz Nominierung durch die Partei zunächst Repräsentanten der Nation, nicht der Partei ${ }^{51}$. Nur mit Hilfe der Streitkräfte wurde der Konflikt zugunsten von Senghor entschieden ${ }^{52}$. Mamadou Dia wurde verhaftet und 1963 zu lebenslanger Haft verurteilt ${ }^{53}$. Die Bevölkerung blieb gegenüber den Ereignissen weitgehend indifferent, doch erhielt Senghor den bedingungslosen Rückhalt der Marabuts, die über die wachsende Kommerzialisierung der Erdnußernte durch neu gegründete senegalesische Institutionen beunruhigt waren und eine Einschränkung ihrer ökonomischen Privilegien befürchteten ${ }^{54}$.

Die Nationalversammlung beschloß am 18. 12. 1962 mit den Stimmen aller anwesenden Abgeordneten eine Verfassungsänderung, die das Amt des Ministerpräsidenten abschaffte und die Exekutivgewalt allein beim Staatspräsidenten konzentrierte. Nachdem in einem Verfassungsgesetz vom 2. 1. 1963 eine weitere, aller-

48 Gleichzeitig hob er aber die Notwendigkeit einer besseren Organisation der UPS und einer verstärkten

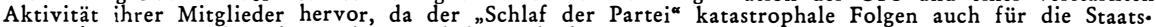
institutionen, vor allem das Parlament, habe; vgl. die Ausführungen Senghors vor dem Conseil National der UPS vom 8. 7. 1962, abgedruckt bei Gerard de Robillard de Beaurepaire: Le régime constitutionnel du Sénégal, Paris (Thèse, Faculté de Droit et des Sciences Economiques) 1964, hekt. (größtenteils nicht paginicrt), Ilème sous-partie, $S .9$.

49 Ebd., S. 4; dort auch das folgende Zitat von Mamadou Dia.

50 Das von Dia zunächst ins Feld geführte, später aber aufgegebene Argument, der Antrag sei deswegen hinfällig, weıl der während des Auseinanderbrechens der Mali-Föderation erlassene und seither immer verlängerte fusuahmezustand noch bestehe, sticht nicht, da nach Art. 41 der Verfassung vom 26. 8 . 1960 die Nationalversammlung während des Ausnahmezustands in ihren Kompetenzen nicht beschnitten war.

51 Robillard de Beaurepaire (Anm. 48), IIème sous-partie, S. 5.

52 Zum Ablauf der Ereignisse im einzelnen siehe: Ernest Milcent: Au carrefour des options africaines: Le Sénégal, Paris 1965, S. $83 \mathrm{ff}$.

531972 wurden die Urteile gegen ihn und einige seiner Mitarbeiter gemildert (für Dia nun insgesamt 20 Jahre Haft), doch lehnt Dia es angeblich ab, das Gefängnıs zu verlassen, solange er nicht voll rehabilitiert ist; vgl. Colin Legum (ed.): Africa Contemporary Record 1972-73, London/New York 1973, B 713.

$54 \mathrm{Zu}$ diesen Angaben im einzelnen und zur Verflechtung der Interessen der Marabuts mit denen ausländischer, besonders französischer, Unternehmen, deren Vertrauen Senghor genoß, siehe Monique Lafon: Eléments sur la crise sénégalaise, in: Economie et Politique (Brüssel), Nr. 103, Februar 1963, S. 87 f. Der Ausbau der Beziehungen zu den osteuropäischen Staaten und eine Reise Mamadou Dias in die UdSSR im August 1962 hatten das Mißtrauen der konservativen Kreise in Senegal noch anwachsen lassen. 
dings geringfügige Änderung der Verfassung vorgenommen worden war ${ }^{55}$, wurde diese einer 'T'otalrevision unterzogen und am 3. 3. 1963 einem Referendum unterworfen.

Senghor betonte bei mehreren Gelegenheiten, Schuld an dem Konflikt zwischen Mamadou Dia und ihm trage zu einem großen Teil die in der bisherigen Verfassung angelegte dualistische Exekutive, die im politisch reifen Europa möglich, der politischen und sozialen Realität Senegals aber nicht angepaßt sei56. In einer Rede an der Universität Straßburg im November 1964 erklärte Senghor, das Wesen der Demokratie liege in der gegenwärtigen Entwicklungsphase in Schwarzafrika, wo der Präsident in seiner Person die Nation verkörpere wie früher der Monarch sein Volk, nicht in einer Teilung der Macht, sondern in der freien Wahl dessen, der sie innehabe ${ }^{57}$. Die Verfassung der „II. Senegalesischen Republik“ brachte dementsprechend die Einführung eines reinen Präsidialsystems und damit eine beträchtliche Verstärkung der Kompetenzen des Präsidenten der Republik. Ihm wurden nun in Analogie zu Art. 16 der Verfassung der V. Französischen Republik extensive Notstandsvollmachten zugestanden, er war alleiniger Inhaber der exekutiven Gewalt, war Chef der Streitkräfte und der Verwaltung, besaß gegenüber der Nationalversammlung das Recht zu einem suspensiven Veto, das diese nur mit drei Fünfteln ihrer Mitglieder zurückweisen konnte, u. a. Für die Präsidentschaft kandidieren konnte nach Art. 23 und 24 der Verfassung jeder mindestens 35 Jahre alte senegalesische Staatsbürger, der die Unterschriften von mindestens 50 Wahlberechtigten, darunter 10 Abgeordneten der Nationalversammlung, vorlegte. Da 79 von 80 Abgeordneten der UPS angehörten ${ }^{53}$ und das neue Wahlgesetz zur Nationalversammlung ${ }^{59}$ - außer bei Parteispaltung - gewährleistete, daß nur eine Partei im Parlament vertreten war, wurden damit Oppositionskandidaten von der Bewerbung ausgeschlossen. Der Staatspräsident wurde im Gegensatz zur Verfassung von 1960 in allgemeiner Wahl mit absoluter, ggfs. im zweiten Wahlgang mit relativer Mehrheit auf vier Jahre gewählt. Er war der Nationalversammlung nicht verantwortlich, konnte diese nun aber auch nicht mehr auflösen. Ein verfassungsergänzendes Gesetz über die Zahl der Mitglieder des Parlaments und die Bedingungen für die Wählbarkeit ${ }^{60}$ bekräftigte die strikte Trennung zwischen Exekutive und Legislative und führte nach dem Vorbild der Verfassung der V. Französischen Republik die Inkompatibilität zwischen Ministeramt und Abgeordnetenmandat ein. $\mathrm{Da}$ jedoch die Ministrablen aus Prestigegründen zunächst ebenfalls kandidierten, wurde für die Listen der Parteien neben den 80 Kandidaten eine Ergänzungsliste mit 20 Namen vorgeschrieben. Um regional konzentrierter Opposition (z. B. in Casamance) endgültig die Chancen zu nehmen, aber auch um innerhalb der UPS die Bildung regionaler Hausmachtstellungen $\mathrm{zu}$ erschweren und die nationale Einheit zu betonen, wurde ganz Senegal nun zu einem einzigen Wahlkreis erklärt, in dem die Liste mit der relativen Mehrheit alle 80 Sitze erhielt. Auch die Wahlperiode der gleichzeitig mit dem Staatspräsidenten gewählten Nationalversammlung wurde auf vier Jahre festgelegt.

\footnotetext{
55 Art. 64 und 65: Qualifizierte Niehrheit für die Anklage vor der Haute Cour und deren Zusammensetzung, Loi 63-02 vom 2. 1. 1963, JORS 7. 1. 1963, S. 21

56 Vgl. die Rede Senghors am 1. 3. 1963 in Diourbel, wiedergegeben bei Robillard de Beaurepaire (Anm. 48), Ilème sous-partie, S. 21. Ebd. S. $21 \mathrm{ff}$. weitere Zitate im selben Sinne.

57 Zitiert bei Markovitz (Anm. 4), S. 204.

58 Ein Abgeordzeter war zum BMS übergetreten.

59 Ord. 63-02 vom 6. 6. 1963, JORS 15. 6. 1963, numéro spécial, S. 795

60 Ord. 63-04 voin 6. 6. 1963, ebd. S. 797.
} 
Erstmals in der Praxis relevant wurde ein bereits 1961 eingerichteter Wirtschafts- und Sozialrat ${ }^{61}$ mit beratender Kompetenz, der bis 1963 nie zusammengetreten war. Nach der Verfassung und den neuen Ausführungsbestimmungen gehörten dem Rat 45 Vertreter der verschiedenen Berufsgruppen an ${ }^{62}$, die durch die Gewerkschaften, die betreffenden Berufsorganisationen oder durch den Präsidenten der Republik ausgewählt wurden. Da nicht nur Senegalesen, sondern auch durch Niederlassungskonvention mit diesen gleichgestellte Ausländer Mitglieder des Wirtschafts- und Sozialrates sein können, besitzen vor allem die in der senegalesischen Wirtschaft mächtigen Franzosen in diesem Rat die Möglichkeit, ihre Interessen in einem Verfassungsorgan zu vertreten. Der Wirtschaftsund Sozialrat bietet den verschiedenen sozialen Gruppen somit einen institutionellen Kontakt untereinander und ermöglicht dem Präsidenten der Republik und der Nationalversammlung neben der Konsultierung dieses Gremiums bei entsprechenden Gesetzesvorhaben auch eine, allerdings sehr beschränkte, Kontrolle über die Aktivitäten und Planungen der einzelnen Gruppen. $\mathrm{Da}$ in der Praxis im Wirtschafts- und Sozialrat auch die langfristigen Wirtschaftspläne, wichtige Entwicklungsvorhaben etc. besprochen werden, besitzt er eine seine beratende Funktion übersteigende Bedeutung ${ }^{63}$.

Trotz der Ablehnung des Verfassungsentwurfs durch die Oppositionsparteien, die vor allem den Machtzuwachs für den Staatspräsidenten und den Ausschluß der Opposition von Präsidentschaftskandidaturen kritisierte, wurde die Verfassung mit 1155077 Ja- gegen 6349 Nein-Stimmen angenommen. Bei den Präsidentschaftswahlen am 1. 12. 1963 war L. S. Senghor einziger Kandidat, und bei den gleichzeitig stattfindenden Wahlen zur Nationalversammlung siegte die UPS überlegen gegen eine sich letztmals stellende Oppositionsgruppierung, die sehr heterogene "Démocratie et Unité Sénégalaise" (DUS) ${ }^{64}$, zu der sich die noch bestehenden Oppositionsparteien zusammengeschlossen hatten, die nur in den im Vergleich zum übrigen Senegal wirtschaftlich zurückgebliebenen Regionen Casamance und im Tukulör-Gebiet des Fleuve größere Stimmengewinne erzielen konnten. Neben dem PRA-Sénégal, der einen gewissen Rückhalt in Gewerkschaftskreisen und in Casamance (der Parteiführer Assane Seck war der erste aus Casamance stammende Universitätsabsolvent ${ }^{65}$ ) besaß, auf dem Land aber ohne jeglichen Einfluß war, und dem verbotenen PAI zählten zum Oppositionsbündnis vor allem der 1961 gegründete „Bloc des Masses Sénégalaises“ (BMS), der sich aus früheren PSAS-Mitgliedern und islamischen Notablen rekrutierte ${ }^{66}$. Noch vor den Wahlen von 1963 war es Senghor gelungen, 12 führende Mitglieder des BMS zum Übertritt in die UPS zu bewegen und die Partei damit erheblich zu schwächen ${ }^{67}$. Die Reste des BMS schlossen sich im Juni 1964 mit jungen Technokraten, meist Angehörigen des "Clans“ von Mamadou Dia, zum „Front

61 Loi vom 23. 6. 1961, JORS 10. 7. 1961.

62 Nach den Ausführungsbestimmungen der Ord. vom 4. 7. 1963 (JORS 20. 7. 1963) und des Dekretes vom 3. 7. 1963 (JORS 24. 8. 1963) setzt sich der Rat zusammen aus je neun Vertretern 1. der Arbeitnehmer des öffentlichen und privaten Bereichs, 2. von Banken, Handel und Handwerk, 3. der Industrie und des Bergbaus, 4. der Landwirtschaft und 5. neun Personen, die aufgrund ihrer fachlichen Qualifikation ausgewd่hlt werden. Bei Drittelerneuerung nach zwei Jahren beträgt die Mandatsdauer sechs Jahre, Wiederwahl ist möglich.

63 Lavroff (Anm. 35), S. 177 f.

641122838 UPS- gegen 66227 DUS-Stimmen; Ergebnis nach Wahlkreisen bei Lô (Anm. 9), S. 205.

65 Traoré (Anm. 32 bzw. 9), S. 92 f.

66 Eine Analyse der Oppositionsgruppen liefern Ismaila Diop: Les élections présidentielles et législatives du ler décenbre 1963 au Sénégail, Paris (Mémoire DES Science Politique, Faculté de Droit et des Sciences Economiques) 1964 (masch.), S. 53 ff. und Traoré (Anm. 32 bzw. 9), S. 92 ff.

67 Traoré, ebd., S. 90. 
National Sénégalais" (FNS) unter Cheikh Anta Diop zusammen, der von der Regierung im Oktober 1964 ohne Begründung aufgelöst wurde, vermutlich, weil das Risiko, daß Anhänger Mamadou Dias einen Kristallisationspunkt bildeten, noch zu groß schien. Nachdem sich im Juni 1966 auch der PRA-Sénégal der UPS anschloß und seine zeitweilig inhaftierten Führer Assane Seck und Abdoulaye Ly in die Regierung eintraten ${ }^{63}$, besteht als einzige legal zugelassene Partei nur noch die UPS. Die Verfassung geht zwar in Art. 3 von einem Parteienpluralismus aus, und die Neugründung politischer Parteien ist juristisch möglich; in Ausführung eines Verfassungsauftrags wurde 1964 ein aus fünf Artikeln bestehendes Parteiengeset $\mathrm{z}^{69}$ erlassen, das in vagen Formulierungen vorschreibt, die Tätigkeit der Parteien müsse sich im gesetzlichen Rahmen bewegen, dürfe nicht gegen die Demokratie gerichtet sein und nicht vom Ausland unterstützt werden. Jede Partei erhält vom Innenminister eine Bestätigung ihrer Zulassung und ist bei Strafe der Auflösung gehalten, jährlich die Namen ihrer Funktionäre bekanntzugeben und einen Rechenschaftsbericht über ihre Finanzen vorzulegen. Bis heute wurde jedoch keine neue politische Partei genehmigt ${ }^{70}$.

Mit der Aufnahme des PRA-Sénégal in die UPS endete 1966 auch die Tätigkeit der letzten Partei, die wenigstens außerhalb des Parlaments legal eine Opposition zur Regierung hatte bilden können. Die Bedeutung der UPS innerhalb des Staates wurde dadurch noch mehr unterstrichen, und einige Besonderheiten des politischen Systems aufgrund der faktischen Einpartei-Herrschaft (wie die Einräumung eines für die alte PRA-Führung sehr günstigen Proporzes nicht nur in den UPS-Führungsgremien, sondern auch in Regierung und Parlament ${ }^{71}$ ) waren nun noch weniger als zuvor aus dem Verfassungstext allein zu entnehmen. Die Konzentration der Ämter des Staats- und Parteichefs in der Hand von L. S. Senghor verstärkten noch die durch die Verfassungsreform von 1963 ohnehin angewachsene Macht der Exekutive und ließen in gleichem Maße die Bedeutung des Parlaments innerhalb des Verfassungssystems zurückgehen. Obwohl die Gesetzesinitiative laut Verfassung sowohl dem Präsidenten der Republik als auch der Nationalversammlung zusteht, hat das Parlament zuletzt 1963 und 1964 vier (von damals insgesamt 122) Gesetzen verabschiedet, die auf seine eigene Initiative zurückgingen $^{72}$. Auch die Kontrollfunktion der Nationalversammlung wurde nicht nur durch den Entzug des Mißtrauensvotums gegen die Regierung beschnitten. Durch die Symbiose von UPS und Staatsapparat, die in der Terminologie der Partei als „Zusammenarbeit" bezeichnet wird, werden die allgemeinen Richtlinien der Politik vom Kongreß der Partei und dem vom Kongreß gewählten Nationalrat der UPS festgelegt, die dauernde Kontrolle über die Ausführung der Richtlinien in der Praxis liegt jedoch beim vom Nationalrat gewählten Comité central bzw. dem von diesem aus seiner Mitte bestellten, 21 Mitglieder zählenden Bureau politique der Partei und erst a posteriori bei Nationalversammlung und Partei-

$68 \mathrm{Zu}$ den Verhandlungen im einzelnen siehe Zuccarelli (Anm. 12), S. 99 ff.

69 Gesetz vom 24. 1. 1964, JORS vom 19. 3. 1964.

70 Wie weit ernsthafte Versuche unternommen wurden, eine neue Partei zu gründen, ist nur sehr schwer zu ermitteln.

71 Nach Zuccarelli (Anm. 12), S. 102, betrugen die aufgrund des Parteiengesetzes für 1965 mitgeteilten Mitgliederzahlen für den PRA 23 191, für die UPS 414158.

72 Philippe Georges: La procédure législative au Sénégal, in: Annales africaines 1965, S. 173 ff. und Zuccarelli (Anm. 12), S. 275. Z. T. ist diese Entwicklung sicher auch auf die auch in anderen Staaten zu beobachtende Tendenz des Rückgangs von Gesetzesinitiativen aus den Reihen des Parlaments zurückzuführen, da die Exekutive in Senegal wie anderswo über qualifizierteres Personal zur Ausarbeitung von Gesetzentwürfen verfügt. 
kongreß ${ }^{73}$. Als eigentliches Machtzentrum, das die wichtigsten Entscheidungen fällt und ihre Ausführung kontrolliert, kristallisiert sich damit das Comité central der IJPS bzw. das Bureau politique heraus. Alle wichtigen Entscheidungen werden jedoch vom einzigen bedeutenden Theoretiker der Partei, Staats- und Parteichef L. S. Senghor, getroffen; sämtliche Parteiinstanzen beschränken sich auf rein formelle Zustimmung oder unbedeutende Detailänderungen an seinen Vorlagen.

Eine wesentiiche Schwäche der Partei liegt in ihrer mangelnden organisatorischen Verfestigung, die sie eher als Kader- denn als Massenpartei ausweist. Da die Zahl der verkauften Mitgliedskarten über die quantitative Vertretung einer Section auf dem laut Satzung alle zwei Jahre stattfinden Parteikongreß entscheidet, kommt es vor, daß Parteiführer eine größere Anzahl von Karten kaufen, diese unter ihrer lokalen Klientel verteilen und auf dem Kongreß ein entsprechend starkes Stimmengewicht besitzen. Die offizielle Mitgliederzahl der UPS ist so beträchtlichen Schwankungen unterworfen ${ }^{74}$. Eine Unterdrückung des ClanKampfes innerhalb der Partei und damit eine erwünschte Stärkung des Zentrums der UPS und ihrer Effizienz würde jedoch zu einem Verlust an Flexibilität gegenüber Krisensituationen auf lokaler Ebene führen, was häufig eine ambivalente Strategie der zentralen politischen Instanzen gegenüber der Peripherie zur Folge hat ${ }^{75}$.

Senghor betont weiterhin die strikte Trennung von Partei und Staat und sieht in der UPS nur das ausführende Organ des Staates. So arbeiten Verwaltungsund Parteiapparat formal weiter völlig unabhängig voneinander. Die Bürokraten, die zu Beginn der sechziger Jahre zwar zum "Clan“ von Mamadou Dia gezählt werden konnten, Aufstiegschancen jedoch primär in der Verwaltung suchten, haben sich unter Senghor stärker der Parteipolitik zugewandt und suchen nun in größerem Maße Zugang zu politischen Ämtern ${ }^{76}$. Auch die Justiz ist unabhängig von der Exekutive. Zum Beleg für die Einhaltung rechtsstaatlicher Normen verweist Zuccarelli77 auf ein Beispiel Ende der sechziger Jahre, als die Regierung einen Gesetzesentwurf zurückzog, den die Cour Suprême in einem - die Regierung nicht bindenden - Gutachten als nicht konform mit der in der Verfassung garantierten Versammlungsfreiheit bezeichnete; andererseits scheinen im Prozeß gegen Mamadou Dia und seine Anhänger die Grenzen zwischen einem formal nach rechtsstaatlichen Prinzipien abgehaltenen Verfahren, für das Senghor offenbar plädierte, und einem politischen Prozeß, den eine Anzahl von Dia-Gegnern forderte, fließend gewesen zu $\operatorname{sein}^{78}$.

\footnotetext{
73 Siehe Zuccarelli (Anm. 12), S. 284. Auf dem 8. UPS-Parteikongreß vom 16. bis $18.12 . \quad 1972$ wurden einige Parteiinstitutionen imbenannt; das Comité central hieß bisher Bureau politique, und anstelle des jetzigen Bureau politique bestand ein politisches Sekretariat. Laut UPS-Satzung tagt das Bureau politique wöchentlich, das Comité central monatlich und der Conseil national jährlich

74 1962: 289 938, 1963 (Parteitag vor Parlaments- und Präsidentschaftswahlen): 467 732, 1964: 292 509, 1965: 418 228, 1966: 385 093, 1967 (Parteitag vor den Parlaments- und Präsidentschaftswahlen von 1968) 540 814; Zahlen nach Zuccarelli (Anm. 12), S. 125, der nur für 1965 die Erhöhung der Mitgliederzahl auf verstärkte Arbeit der Parteiorgane zurückführt. Für März 1972 wurde die Zahl der Parteimitglieder auf ïber 700000 angegeben, wobei 1972 (Parteitag vor den Wahlen vom Januar 1973 im Dezember 1972) 150000 neue Mitgliedskarten ausgegeben wurden, vgl. Colin Legum (Anm. 53), B 711.

75 Clement Cottingham: Political Consolidation and Centre-Local Relations in Senegal, in: Canadian Journal of African Studies, Bd. 4, Nr. 1, Winter 1970, S. $101 \mathrm{ff}$

6 Adamolekun (Anm. 47), S. 554; S. 547, Tabelle 1 verdeutlicht Adamolekun, daß sich die Zahl der Bürokraten in der Regierung unter Senghor gegenüber Dia verdoppelt hat.

77 Zuccarelli (Anm. 12), S. 290.

78 Vgl. u. a. Paquet (Anm. 16), S. $115 \mathrm{ff}$.
} 
IV.

1967 wurde auf Initiative Senghors die Verfassung in einigen Punkten geändert, wobei der Staatspräsident, dem nach der Verfassung die alleinige Entscheidung darüber zusteht, ob eine Verfassungsänderung nur durch die Abstimmung in der Nationalversammlung oder durch Referendum ratifiziert werden soll, wenige Monate vor den fälligen Neuwahlen auf ein Referendum verzichtete ${ }^{79}$. Die Position des Staatspräsidenten wurde insofern gestärkt, als er außer der Erhöhung der Dauer seines Mandats von vier auf fünf Jahre ${ }^{80}$ auch das Recht erhielt, die Nationalversammlung aufzulösen. Damit wurde eine wesentliche Komponente des Gleichgewichts zwischen Exekutive und Legislative nach dem „klassischen“ Präsidialsystem aufgegeben, zumal der Präsident vom Parlament weiterhin nicht gestürzt werden konnte. Er konnte die Auflösung jedoch erst nach Ablauf von drei Jahren der laufenden Wahlperiode aussprechen, aber auch dann nicht im Falle von Notstandsvollmachten für den Präsidenten (die in der Neufassung von Art. 47 erheblich eingeschränkt wurden). Löste der Präsident jedoch das Parlament auf, mußte er zugleich auch sein Amt zur Disposition stellen, so daß in letzter Instanz die Wählerschaft über den Konflikt zwischen Exekutive und Legislative zu entscheiden hatte. Für die Zeit nach den nächsten Präsidentschaftswahlen (also erstmals 1973) gilt die Änderung der vielfach kritisierten Voraussetzungen zur Präsidentschaftskandidatur. Wählbar ist jetzt jeder Bewerber, der von einer offiziell zugelassenen Partei nominiert wird oder die Unterschriften von 50 Wahlberechtigten, darunter 10 Abgeordneten, vorweisen kann. Solange allerdings nur die UPS als legal konstituierte Partei besteht, ändert sich an deren faktischem Monopol für die Nominierung des Präsidenten der Republik nichts. Die Verfassungsreform hob ferner die Inkompatibilität zwischen Ministeramt und Abgeordnetenmandat auf; entsprechend wurde in einer Anderung des Wahlgesetzes die Reserveliste der 20 Sitze gestrichen ${ }^{81}$. Jean-Claude Gautron weist zu Recht darauf hin, daß die offizielle Begründung für die Aufhebung der Inkompatibilität, die Beseitigung der Reserveliste, nur die Folgeerscheinung der neuen Regelung ist; daß in der Substanz jedoch das Parlament noch stärker an die Exekutive gebunden wird und der Staatspräsident größere Handlungsfreiheit bei einer Regierungsumbildung hat, da die ausscheidenden Minister über die Nationalversammlung auf hoher Ebene dem politischen Leben intregriert bleiben ${ }^{82}$. Zugleich bedeutete die neue Regelung einen Rückschlag für die Politiker in den "Clans“, deren „politique politicienne“ (d. h. Intrigen, um persönliche Macht zu erwerben) von Senghor bei zahlreichen Anlässen kritisiert worden war und die nun neben einer wachsenden $Z$ ahl von Technokraten in der Regierung auch hinnehmen mußten, daß diese leichteren Zugang zur Nationalversammlung hatten ${ }^{83}$.

Das plötzliche Aufbrechen der Krise in Senegal, für das die Nachricht von den Unruhen in Paris nur der auslösende Funke war, hatte langfristig angelegte sozioökonomische und politische Gründe. Während die Bevölkerung jährlich um über $2 \%$ zunahm, stagnierte die Erdnuß-Produktion bzw. war aufgrund

79 Zum technischen Ablauf der Vorarbeiten siehe Jean-Claude Gautron: La révision constitutionnelle du 20 juin 1967 au Sénégal, in: Revue Sénégalaise de Droit, Nr. 2, Dezember 1967, S. 9, Anm. 8.

80 Die Wahlperiode des Parlaments wurde ebenfalls auf fünf Jahre ausgedehnt.

81 Loi organique vom: 8. 7. 1967, JORS 10. 7. 1967.

82 Gautron (Anm. 79), S. 14. 
von Trockenheit 1967 sogar rückläufig gewesen. Gleichzeitig waren die Preise für Erdnuß auf dem Weltmarkt und damit das durchschnittliche Pro-Kopf-Einkommen der Bevölkerung gesunken. Mit dem hohen Anwachsen der Stadtbevölkerung (vor allem in Dakar) hatte die Zunahme der Arbeitsplätze bei weitem nicht Schritt halten können ${ }^{84}$. 1960-1966 betrug die Steigerung der Lebensmittelpreise in den Städten $33 \%$, doch waren die Gehälter im Zuge einer AusterityPolitik seit 1963 blockiert. Der tertiäre Bereich, insbesondere der Staatsapparat, war für Neurekrutierungen geschlossen ${ }^{85}$, so daß die Arbeitslosigkeit weiter gewachsen war $^{86}$. $\mathrm{Zu}$ dieser vor allem von den Gewerkschaften heftig kritisierten Entwicklung kam eine unter den Intellektuellen, besonders den Studenten an der Universität Dakar, verbreitete Unzufriedenheit über Mängel im Staats- und Parteiapparat, fehlende Aufstiegschancen und den Führungsstil Senghors, dessen Art, wichtige Beschlüsse allein zu fassen, durch die Verfassungsreform von 1967 noch augenfälliger geworden war.

Senghor kündigte nach den Unruhen noch 1968 Konsequenzen in Form einer Verfassungsreform an, die die Regierung durch „Dekonzentration“ effizienter machen sollte. Nachdem auf einer Studientagung der UPS im Mai 1969 grundsätzliche Úbereinstimmung über die Prinzipien der Verfassungsreform erzielt und diese von der Nationalversammlung im Dezember 1969 gebilligt worden war, wählte Senghor für die Ratifizierung, der diesmal aufgrund der vorausgegangenen Unruhen besonderes Gewicht zukam, die "feierliche" Form des Referendums, das am 22. 2. 1970 bei $95 \%$ Abstimmungsbeteiligung und 1261580 Ja- gegen 542 Nein-Stimmen wenigstens nach außen einen allgemeinen Konsens über die Verfassung in ihrer geänderten Form zeigte ${ }^{37}$. Wichtigste Neuerung war die Wiedereinführung des Amtes des Premierministers, die Senghor damit begründete, daß das Fehlen parlamentarischer Verantwortlichkeit der Regierung in der Verfassung von 1963 die Minister dazu geführt habe, Verantwortung von sich auf andere abzuwälzen (System des „ponce-pilatisme“"88). Die Stellung des Premierministers ist jedoch erheblich schwächer als unter dem System der Verfassung vom 26. 8. 1960. Er wird vom Präsidenten der Republik ernannt und kann sein Amt sowohl durch Mißtrauensvotum des Parlaments als auch durch Entlassung durch den Staatspräsidenten verlieren. Der Präsident ernennt auf Vorschlag des Regierungschefs auch die Minister, kann diese aber auch auf eigene Initiative abberufen. Die Regierung hat die Politik auszuführen, die der Präsident der Republik festlegt. Durch diese Verfassungsbestimmungen ist gesichert, daß der Premierminister dem Präsidenten der Republik eindeutig untergeordnet ist und

83 Vgl. Adamolekun (Anm. 47), S. 555, der es für möglich hält, daß die Wiedereinführung der Inkompatibilitätsbestimmungen 1970 auf den Druck der "professionellen “ Politiker zurückgeht.

$84 \mathrm{Zu}$ diesen $\Lambda$ ngaben siehe. J. Roch/G. Rocheteau: Economie et Population. Le cas du Sénégal, in: Cahiers O.R.S.T.O.M. (Paris), Série Sciences humaines, Bd. 8, Nr. 1, 1971, S. 63.

85 François Zuccarelli: Les élections présidentielles et légıslatives du 25 février 1968 au Sénégal, Montpellier (Mémoire DES, Faculté de Droit et des Sciences Economiques) 1969 (hekt.), S. 8.

86 Im März 1969 betrug die Zahl der Personen, die sich offiziell als arbeitslos hatten registrieren lassen 43 626, davon 83,5 Prozent in der Region des Cap Vert (Dakar); die tatsächliche Zahl dürfte jedoch erheblich höher liegen. Angaben nach: Marchés Tropicaux et Méditerranéens 13.6 .1970 ,
S. 1842.

87 Die Zahlen für die Beteiligung an der Abstimmung liegen mit Sicherheit zu hoch; ein französischer Beobachter, der in einem Stimmlokal in einer ländlichen Gegend im Norden Senegals die Abstimmung verfolgte, gibt an, daß statt der offiziell gemeldeten 99 Prozent die Abstimmungsbeteiligung stimmung verfolgte, gibt an, daß statt der offiziell gemeldeten 99 Prozent die Abstimmungsbeteiligung ca. 40-50 Prozent betragen habe (persönliche Information); für die Wahlen von 1968 schätzt Zuc-
carelli (Anm. 85), S. 80, daß die tatsächliche Wahlbeteiligung statt der offiziell angegebenen 94,5 Prozent etwa 65-75 Prozent betragen hat.

88 Von Senghor geprägter Begriff, zitiert u. a. bei Said Michel Ajami: Réflexions sur la ndéconcentration de l'exécutif “ au Sénégal après la révision constitutionnelle de 1970, in: Revue Juridique et Politique 25, 1971 , S. 251. 
Machtproben wie 1962 durch die Verfassungskonstruktion ausgeschlossen sind. Das neue Verfassungssystem führt nach Senghor keine dualistische Exekutive ein, vielmehr spricht er von einem "régime présidentiel déconcentré" ${ }^{89}$. Neugestaltet wurde auch das Recht des Präsidenten der Republik, das Parlament aufzulösen. Nur in dem Fall, in dem die Nationalversammlung die Regierung stürzt, kann der Staatspräsident das Parlament auflösen, und zwar ohne Rückwirkung auf sein eigenes Mandat, das wie das der Nationalversammlung fünf Jahre beträgt. Allerdings kann der Präsident der Republik erst ein Jahr nach einer durch Parlamentsauflösung bedingten Neuwahl die Nationalversammlung erneut auflösen. Konnte sich der Staatspräsident nach der bisherigen Verfassung nach Ablauf seiner Amtszeit beliebig oft erneut kandidieren, so darf er sich nach der Verfassungsreform von 1970 nur einmal zur Wiederwahl stellen, was für Senghor jedoch eine Kandidatur auch für 1978 nicht ausschließt, da nach Art. 3 des die Verfassung ändernden Gesetzes vom 26. 2. $1970^{90}$ die Wahlen vor der Einführung der Verfassungsreform nicht in Rechnung gestellt werden.

Auffallendster Zug der Verfassungsreform von 1970 ist somit die Einführung einer Regierung, deren Stellung gegenüber dem Präsidenten der Republik wie der Nationalversammlung so schwach ist, daß sie im Falle von Krisen wie 1968 ohne Schwierigkeit ausgewechselt werden kann. Das Regime hat dadurch an Flexibilität gewonnen und gleichzeitig den Präsidenten der Republik dem Bereich unmittelbarer Kritik entzogen, da für die Ausführung der konkreten Politik zunächst der Premierminister verantwortlich gemacht werden kann. Die Autorität des Staatspräsidenten war seit Mitte der sechziger Jahre gesunken, seit Senghor direkt und für die breite Bevölkerung auf Rundreisen durch Senegal als Chef der Exekutive auch sichtbar mit der Regierungsführung befaßt war und die wirtschaftlichen Rückschläge unmittelbar ihm zur Last gelegt werden konnten. Der Staatspräsident, der jedes Jahr mehrere Monate im Ausland verbringt, ist durch die Reformen in der Führung der Regierungsarbeit entlastet, behält sich aber weiter entscheidende Bereiche wie die Formulierung der Theorie der Partei und der Außenpolitik vor. Dagegen besitzt der Premierminister Handlungsfreiheit vor allem auf wirtschaftlichem Gebiet. Mit Abdou Diouf, Jahrgang 1935, hat Senghor einen jungen Technokraten zum Regierungschef ernannt, der das Vertrauen der Hochschulabsolventen besitzt. Diouf, der in der UPS bis 1968 keine Rolle gespielt hatte, war seit 1963 in hohen Funktionen im Präsidialamt gewesen, hatte dort wesentlichen Anteil an der Koordinierung der Regierungsarbeit ${ }^{91}$ und war 1968 als Planungs- und Industrieminister ins Kabinett eingetreten. Im Dezember 1972 wurde er zum ersten stellvertretenden Generalsekretär der UPS gewählt.

Ėrgänzt wurden die Verfassungsänderungen 1972 durch eine Modifizierung der Loi organique über die Zahl der Abgeordneten, deren Zahl von 80 auf 100 erhöht wurde ${ }^{92}$, was einem größeren Kreis von clans und pressure groups die Befriedigung ihrer Forderungen erleichtert. Der Unzufriedenheit breiter Kreise der Bevölkerung wurde dadurch Rechnung getragen, daß nur noch 35 der bisherigen 80

89 Siehe ebd.

90 Loi organique $70-15$ vom 26. 2. 1970, JORS 28. 2. 1970, S. 230; Erratum dazu JORS 21. 3. 1970, S. 278. Text abgedruckt u. a. in: Annales Africaines 1969, S. 23-28; Revue Juridique et Politique 25,

Nach Ibrahima Fall: La réforme constitutionnelle du 22 février 1970 au Sénégal, Penant 1971, S. 114, hat Diouf die Funktionen des Premierministers faktisch bereits vor 1970 ausgeübt, die formale Ernennung zum Regierungschef bedeute daher im wesentlichen, daß er für sein Amt nun auch verfassungsrechtlich gegenüber Parlament und Staatspräsident rechenschaftspflichtig sei.

92 Loi organique $72-88$ vom 19.8. 1972, JORS 28. 10. 1972, numéro spécial, S. 1781. 
Abgeordneten wieder nominiert wurden (1968 waren es 52 von 80 gewesen). Ferner erhielt auf dem UPS-Kongreß vom Dezember 1972 eine größere Zahl von Politikern der jüngeren Generation Zugang zu hohen Führungspositionen in der Partei.

V.

Änderungen im institutionellen Bereich der Verfassung waren in Senegal seit der Unabhängigkeit in der Regel die Konsequenz vorausgegangener politischer Krisen, deren Ursachen durch die Verfassungsreformen jedoch nicht beseitigt werden konnten. Dem Zentrum der politischen Macht ist es bis jetzt nicht gelungen, die Kontrolle über wichtige Bereiche des sozioökonomischen Systems zu gewinnen. Das seit der Unabhängigkeit mehrfach veränderte System der landwirtschaftlichen Genossenschaften hat sich als zu komplex erwiesen, um befriedigend zu arbeiten. Als weiterer Störfaktor machen sich auf lokaler Ebene die „clans politiques“ bemerkbar, die die auf ökonomischem Gebiet errichteten „sozialistischen“ Institutionen zur persönlichen Einflußnahme mißbrauchen ${ }^{93}$. Die Überwindung der ErdnußMonokultur ist nicht im erhofften Umfang gelungen; nur 57 Prozent der Einfuhren konnten 1971 durch Exporte abgedeckt werden ${ }^{94}$. Die wirtschaftliche Abhängigkeit von Frankreich ist zwar geringer als zu Beginn der sechziger Jahre, aber immer noch extrem stark ${ }^{95}$. Der direkte Einfluß der Franzosen im politischen Bereich ist etwas zurückgegangen, aber auch nach der Kabinettsumbildung im Anschluß an die Neuwahlen blieb Jean Collin (formell senegalesischer Staatsbürger) im Frühjahr 1973 Innenminister. Innerhalb der UPS-Hierarchie war er 1972 zum Organisationssekretär der Partei aufgestiegen.

Am virulentesten äußert sich eine bis jetzt allerdings nicht organisierte Opposition gegen das etablierte Regime bei den Gewerkschaften und den jungen Intellektuellen, während die Marabuts auf der anderen Seite in den letzten Jahren ebenfalls in größere Distanz zur Partei getreten sind. Die UPS bemüht sich gegenwärtig in verstärktem Maße, die 1969 gegründete „Confédération Nationale des Travailleurs Sénégalais" (CNTS) als der Partei personell und organisatorisch total integrierte Einheitsgewerkschaft $\mathrm{zu}$ etablieren und hat $\mathrm{zu}$ diesem $Z_{\text {weck }}$ selbst die Parteistatuten geändert ${ }^{96}$. Versuche von Führern der aufgelösten UNTS, eine alternative Gewerkschaft zu gründen, wurden unterdrückt, was zu einer noch engeren Aktionseinheit von oppositionellen Gewerkschaftern, unter denen die Lehrer führend sind, Schülern und Studenten geführt hat und eine der Hauptursachen dafür war, daß wenige Wochen nach einem den Zahlen nach erneuten triumphalen Wahlerfolg für Senghor und die Kandidatenliste der UPS zur Nationalversammlung am 28. 1. $1973^{97}$ die Unruhen mit Vehemenz wieder aufbrachen. Die Marabuts,

$93 \mathrm{Vgl}$. Zuccarelli (Anm. 12), S. 257 f.

941970 waren es 79 Prozent gewesen. Aufgrund von Trockenheit hatte Senegal in den letzten Jahren (ausgenonimen 1971/72) eine rückläufige Erdnußproduktion zu verzeichnen, was bei sinkendem Weltmarkt-Preis und fast völliger. Abhängigkeit Senegals vom Erdnußexport nicht nur die privaten Einkommen, sondern auch die staatlichen Einnahmen erheblich reduziert.

951971 importierte Senegal aus Frankreich bei einem Gesamt-Importvolumen von 60,56 Mrd. F CFA Waren im Wert von 28,7 Mrd. F CFA; für den Export betrugen die entsprechenden Zahlen 34,7 Mrd. bzw. 17,97 Mrd. F CFA; vgl.: L'Afrique d'expression française et Madagascar, Europe-Outremer, Mrd. bzw. 17,97 Mrd. F CFA; vgl.: L'Afrique d'expression française et Madagascar, Europe-Outremer,

96 Die CNTS ist a uf allen Ebenen der Partei und des Staates mit ca. 10 Prozent der Mitglieder der jeweiligen Gremien vertreten. Jeder Arbeiter, der UPS-Mitglied ist, gehört obligatorisch der CNTS an, alle CNTS-Funktionäre müssen UPS-Mitglieder sein; vgl. Sénégal: De grandes transformations, in: Europe-Outremer, Nr. 518, März 1973, S. 14.

97 Bei 1399433 Wahlberechtigten stimmten bei einer Wahlbeteiligung von 97,01 Prozent bei den Präsidentschafts- und 96,90 Prozent bei den Parlamentswahlen 99,97 Prozent für Senghor und 99,94 Prozent für die UPS-Liste zur Nationalversammlung; Quelle: Le Soleil (Dakar), Nr. 834, 30. 1. 1973, S. 1. 
auf deren Kooperation die Staats- und Parteiführung aufgrund der Unzufriedenheit in der Bauernschaft angewiesen ist, haben sich zu Mittlern zwischen den ihnen ergebenen Massen und dem zentralen politischen Sektor etablieren können und von der Regierung initiierte soziale, politische und ökonomische Reformen so weit akzeptiert, als sie dadurch ihre eigene Stellung festigen konnten ${ }^{98}$. Seit dem Tode des Mouridenführers Cheikh Falilou M’Backé 1968, der Senghor seit 1945 unterstützt und sich in mehreren für den Staatspräsidenten kritischen Situationen (z. B. Ende 1962) öffentlich hinter ihn gestellt hatte, hat sich das Verhältnis zwischen den islamischen Führern und der Parteifürhrung jedoch verschlechtert. Eine - dann doch unterbliebene - Präsidentschaftskandidatur des Direktors von Air Afrique, Cheikh Fall, der das Vertrauen der Marabuts genießt, schien für die Wahl vom Januar 1973 nicht ausgeschlossen.

Eine Schlüsselrolle fällt in dieser Situation dem Militär (5900 Mann) zu, dessen Führung nach dem Rücktritt von General Jean-Alfred Diallo als Chef der Streitkräfte im Juni 1972 in mehrere Kommandos aufgespalten wurde und somit zunächst neutralisiert scheint. Über die führenden Militärs ist jedoch nur wenig bekannt, so daß die Streitkräfte eine Unbekannte im politischen Kalkül bleiben.

Das politische System Senegals befindet sich gegenwärtig in einer Übergangsphase, die durch ein verstärktes Bemühen der UPS um die Konsolidierung ihrer Macht und durch die Frage der Nachfolge Senghors bestimmt ist. Senghor scheint den Premierminister Abdou Diouf, der seit 1972 sein Stellvertreter in der Partei ist, als Nachfolger zu favorisieren, läßt aber noch offen, ob er 1978 erneut für die Präsidentschaft kandidieren will'99. Die Partei kann, wenn sie größere Kontrolle über ihrem Zugriff bisher nur schwer zugängliche Gruppen wie Gewerkschaften und Marabuts gewinnt, unter neuer Führung verstärkt aus der gegenwärtigen Durchgangsphase hervorgehen. Sollten sich die Oppositionsrichtungen jedoch kräftigen und organisieren können, wäre in der Zeit nach Senghor eine Entwicklung auf ein Mehrparteiensystem hin nicht völlig ausgeschlossen. Die Verfassung, von Senghor immer wieder als oberste Richtschnur für die Spielregeln der politischen Auseinandersetzungen apostrophiert, wäre in ihrer gegenwärtigen Form flexibel genug für eine Reihe von alternativen Entwicklungsmöglichkeiten. Es muß jedoch offenbleiben, ob der bestehende institutionelle Rahmen ausreicht, bei verändertem soziopolitischem Kontext die Macht in einem kontinuierlichen Prozeß auf den oder die Nachfolger Senghors zu übertragen oder ob erneute, vielleicht einschneidende Änderungen der Verfassung erforderlich sind.

98 Vgl. Coulon $(\Lambda \mathrm{nm} .41)$, S. 164

99 Vgl. das Interview Senghors mit Monique Sordet in Europe-Outremer (Anm. 96), S. 9. Dagegen erklärte er Anfang 1974 in mehreren Interviews in der französischen Presse, er hoffe, sich bis 1978 aus der Politik zurückziehen zu können. 
could acceed to citizenship by various methods which by their very nature prevented too many persons from becoming citizens. The main effect of change of status was that the African became thenceforth subject in principle to French law.

There were cases where French law was applicable to non-citizens. For example, when a Frenchman and an African were involved in a dispute, then French law applied. Moreover, parties to a dispute, whether citizens or not, could in some situations agree to submit the matter to the French courts. In situations where customary law did not offer an adequate solution, the courts would apply French law. Customary law could be excluded if its solution offended French notions of public order.

The main effect of the introduction of French law was to interrupt the development of African institutions.

\section{Constitution and Politics in Senegal Since Independence}

\section{By Klaus Ziemer}

Although the same President of the Republic and the same political party have been in power in Senegal since independence in 1960, the political institutions have undergone several fundamental changes. After the 1960 constitution, which introduced a dual executive modelled on the constitution of the Fifth French Republic, the constitution of the "Second Senegalese Republic" concentrated in 1963 all executive power in the hands of the President of the Republic. In contrast, a new constitutional reform in 1960 set up a very week governement in the system of a so-called "pouvoir exécutif déconcentré". The power of the National Assembly was curtailed not only by the text of the constitutions, but above all by the existence of the UPS, single dominant party until 1966, since then the only legally constituted party. All constitutional changes were the results of political crises without being able to abolish their causes. The centre of political power has not yet been able to gain enough control over the peripheral sectors of the political system. As the production of groundnuts has diminished because of drought and the price on the world market has sunk in the last few years, private and public income have fallen, which has caused some unrest among the peasants and reinforced the power of Islamic marabouts who established themselves as a link between the political centre and the masses whom they control. On the other hand, the still only weakly organized UPS tries to integrate the opposition trade unions. Senegal is passing through a period of transition, marked by the efforts of the central political authorities to consolidate their power and to secure a smooth succession of the President of the Republic and General Secretary of the UPS, Léopold Sédar Senghor. 